\title{
Microstructure of aragonite platelets in nacre
}

\author{
C. Kübel***, K. Gries***, R. Kröger****, M. Fritz***, A. Rosenauer*****
}

* Forschungszentrum Karlsruhe, Institute for Nanotechnology, 76344 Eggenstein-Leopoldshafen, Germany

** Fraunhofer IFAM, Wiener Straße 12, 28355 Bremen, Germany

*** University of Bremen, Institute of Biophysics, Otto-Hahn-Allee 1, 28359 Bremen, Germany

**** The University of York, Department of Physics, Heslington, York YO10 5DD, United Kingdom

***** University of Bremen, Institute for Solid State Physics, Otto-Hahn-Allee 1, 28359 Bremen, Germany

Nacre, the inner iridescent layer in mollusk shells, is composed of alternating layers of aragonite and an organic matrix of proteins and $\beta$-chitin. The aragonite, which is a $\mathrm{CaCO}_{3}$ - polymorph, is arranged in polygonal, stacked platelets with a width between $5 \mu \mathrm{m}$ and $10 \mu \mathrm{m}$ and a thickness of about $500 \mathrm{~nm}$. The composite exhibits mechanical properties significantly improved compared to bulk aragonite.

The aragonite platelets show a highly correlated growth with a fairly uniform orientation extending for about 10 to 40 platelets. We investigated the correlated growth of the platelets using a combination of HRTEM (High Resolution Transmission Electron Microscopy), electron diffraction, and electron tomography focusing on the mineral bridges formed between platelets. HRTEM revealed that the mineral bridges consist of quasi single-crystalline aragonite offering the possibility of orientation transfer across these bridges. However, electron tomography showed that not all of the bridges are connecting two platelets, but some are isolated hillocks present on the surface of a platelet (Figure 1).

Surprisingly, the aragonite platelets exhibit a large number of 'voids' with a diameter of up to $30 \mathrm{~nm}$ distributed randomly throughout the platelets. An EELS and EDX analysis suggests.that the 'voids' are filled with an organic material (Figure 2) presumably determining the faceting observed for the 'voids'. Electron tomography shows that the larger 'voids' exhibit a fairly uniform faceting, which can be idealized as dodecahedral shape (Figure 3).

References

[1] K. Gries, et al., Ultramicroscopy, 109 (2009), accepted.

[2] K. Gries, et al., Acta Biomat. (2009), submitted. 

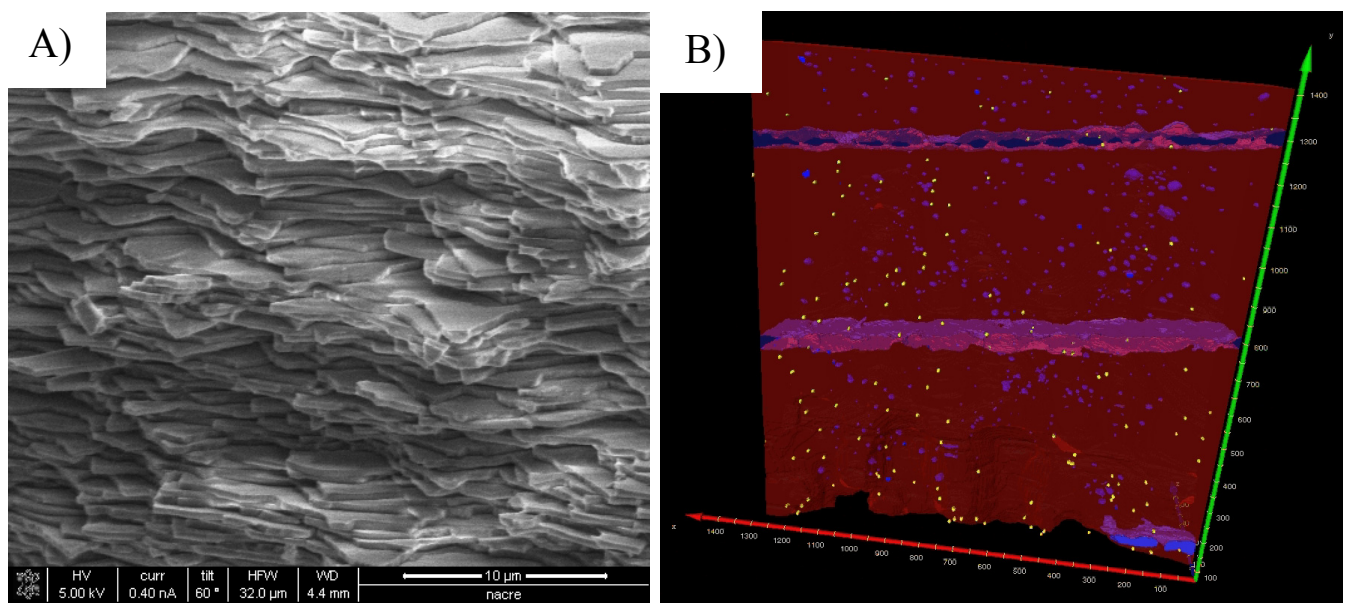

FIG. 1. A. SEM image and B. 3D tomographic reconstruction (aragonite - red; organic matrix blue; gold markers at surface - yellow) of aragonite platelets in nacre.

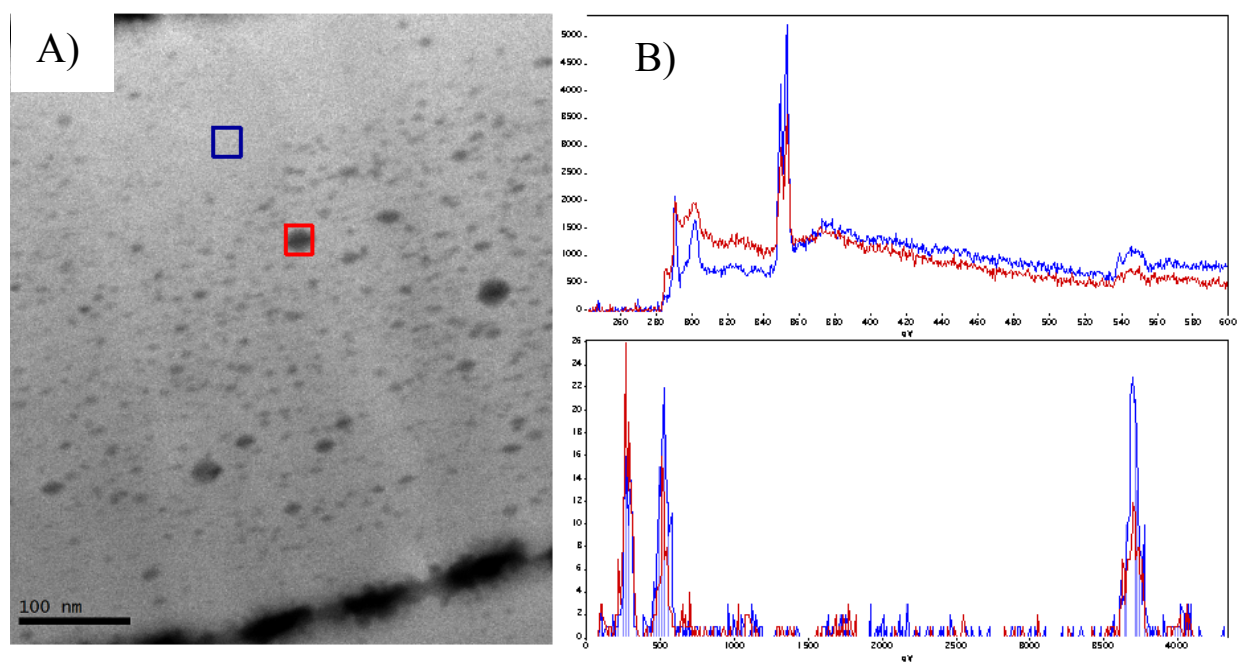

FIG. 2. A. EELS and B. EDX analysis of 'voids' inside the aragonite platelets in nacre.
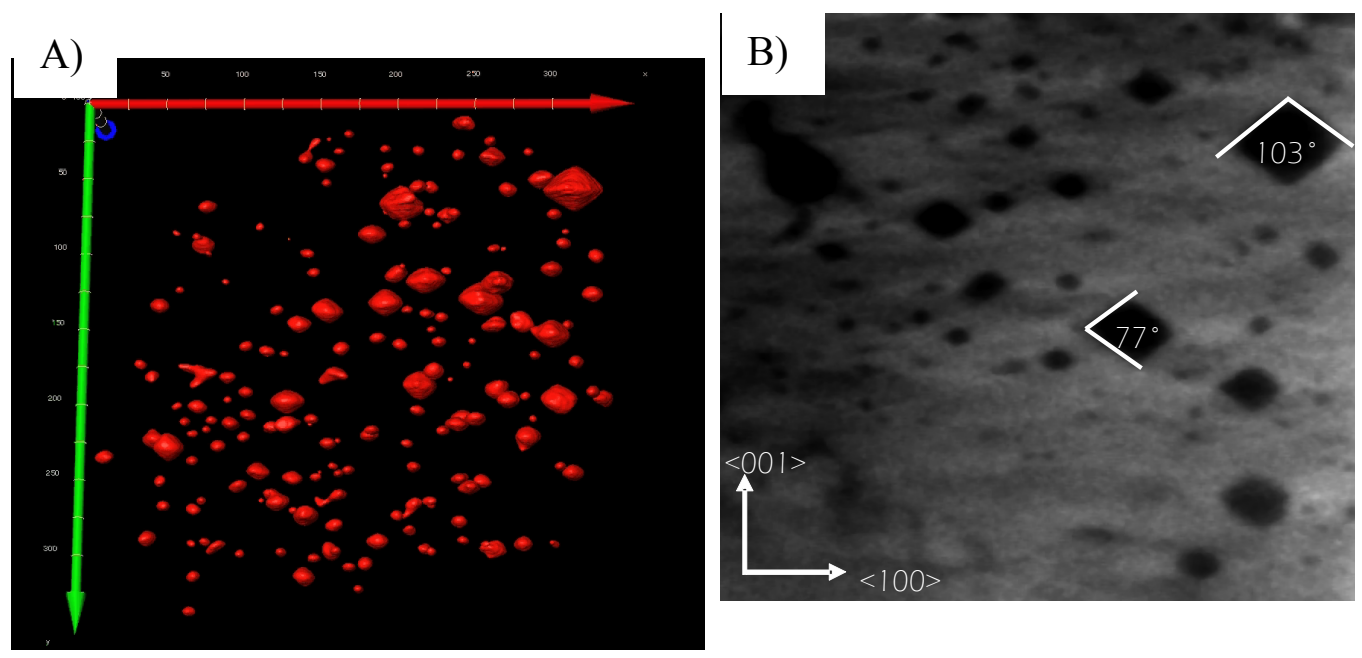

C)

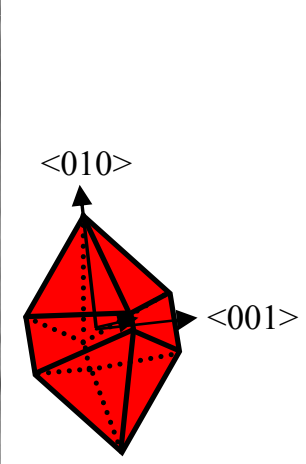

FIG. 3. A. Surface rendering and B. digital slice of a tomographic reconstruction revealing the shape of the "voids'. C. Model for the "voids" with indicated indices. 